\title{
THE USE OF TWINS IN THE ANALYSIS OF ASSORTATIVE MATING
}

\author{
LINDON EAVES \\ Department of Experimental Psychology, University of Oxford
}

Received 11.vii.79

\begin{abstract}
SUMmaRY
The simulations illustrated show that a plausible model for mate selection can generate data on the similarity of twins and their spouses which are remarkably consistent with a transitive model for the effects of mate selection. That is, biological considerations impose constraints upon the relative values of correlations which are not foreseen, for example, by the some advocates of conventional path models although they might be predicted by common sense. In particular, the correlation between the spouses of twins is expected to be non-zero under a model of phenotypic assortment and turns out to be approximately equal to the product of the twin correlation and the square of the marital correlation. The relative magnitudes of the correlations derived from an empirical study of such relationships should enable models of phenotypic assortment to be tested more rigorously. Including both identical and nonidentical twins in the sample studied should permit the inherited and cultural components of the mating system to be identified with more conviction. In the event of one sex playing a more significant role in mate selection for particular traits, such studies should reveal diagnostic patterns of familial correlations as long as male and female twins and their spouses are analysed separately. If the analysis is restricted to phenotypic correlations of the parents, the qualitative findings do not appear to be greatly affected by selection due to assortative mating although a reduction in variance is to be expected if a large proportion of individuals is unable to mate. In such cases twins will also be significantly concordant for mating. The consequences of such varied regimes of assortation for the population structure and the relationship between traits in subsequent generations remain the object of future inquiry.
\end{abstract}

\section{INTRODUGTION}

THE specification of models for the effects of assortative mating on continuous human variation has a long history, but their application to real data has been fairly limited because of the difficulty of disentangling the inherited and cultural components of assortation. Thus, although Fisher (1918) recognised that the primary association between spouses might be either genotypic or phenotypic and specified models for the similarity of relatives accordingly, it is fairly difficult in practice to decide analytically which of the alternatives is appropriate for particular human traits. The matter is further complicated if we recognise with Morton and his associates (Rao, Morton and Yee, 1976) that the primary cause of phenotypic correlation between spouses might be the social environment which influences behavioural development. Unfortunately, in the absence of an analytical design for the resolution of such effects it becomes difficult to do more than interpret particular sets of data in the light of one or other model. Actual discrimination between alternatives is likely to be poor.

The biometrical models of Fisher and the path models of Rao et al. (1976) 
have assumed that assortation is conducted without selection. Wilson (1973) observed that this may not be true and showed how a reduction in variance would follow from stabilising selection imposed as a result of assortative mating.

Generally, such models as have been proposed assume a mechanism of assortation which is "symmetric", that is males and females are looking for the same trait in their spouses. As far as intelligence is concerned, it is assumed that the trait is as important for males choosing females as it is for females choosing males. This need not be the case. It is often suggested that males expect quite different qualities in females from those expected by females in males.

In this paper the consequences for the similarity between spouses of such mechanisms of assortation are examined by computer simulation in an attempt to suggest studies to assist a better understanding of the genetic and social basis of the human mating system.

\section{THE PROPOSED DESIGN}

The study of the phenotypes of identical and fraternal twins has long been recognised as an effective, if fallible, approach to the analysis of the causes of human differences. Studies of morphological and behavioural characteristics of twins are so numerous as to defy review. In general, however, it has been found that the correlation of $\mathrm{MZ}$ twins exceeds that for $\mathrm{DZ}$ twins for a broad spectrum of traits in the physical and behavioural domains suggesting, as a good working hypothesis, that genetical factors affect human behaviour as much as they do any other aspect of human and infra-human variation.

Occasionally, as in the case of social attitudes (Eaves et al., 1978) it is clear that a simple genetic mechanism is insufficient to account for all the similarity of twins and it becomes necessary to invoke the influences of the family or cultural environment, possibly in combination with the genetic effects of the mating system.

The twin study can be extended very simply to provide a similar causal analysis of assortative mating by securing, in addition to observations on twins themselves, data on their spouses. If data are also available on the offspring of twins it is also possible to detect the contribution of the maternal genotype to the shared environment of siblings (e.g., Nance and Corey, 1976). Here we are concerned only with the mechanism of assortation.

It is proposed to ascertain pairs of twins initially and then to ascertain spouses of those twins who have married. The phenotypes of the twins' spouses are then used as indices of the mating preferences of the twins. If these are genetically determined, it is expected that the spouses of $M Z$ twins will correlate more highly than those of $\mathrm{DZ}$ twins. If mating preferences are primarily social, the spouses of twins will correlate to the same extent regardless of zygosity. If selection of spouses depends on factors which are unique to individual twins then the spouses of twins ought to be uncorrelated with one another even though there may be a marital correlation between twins and their spouses. In the event of male and female expectations being different, it is conceivable that the pattern of correlation between twins' spouses will depend on the sex of the twins chosen for study.

In every case, therefore, the findings for the spouses of twins are expected to reveal the mechanism underlying the assortation in exactly the same way 
that the twin method can be exploited to obtain estimates of the genetic and cultural components of any other aspect of the phenotype.

It will be shown by computer simulation that the most appropriate models for mate selection allow the correlation of spouses to be transitive, that is, the correlation of twins' spouses is a function of the marital correlation and the correlation between twins for the mating preferences. Although models which do not enforce transitiveness are mathematically feasible, they do not appear to have any real biological basis.

\section{The MOdel}

It is assumed that a mating can be represented by two processes-the encounter and the decision to mate. It is assumed that encounters are random with respect to phenotype and that a mating will follow an encounter with a probability which depends on the difference between the phenotypes of the potential mates. We define $r$, a uniform random variate and assume that mating will occur if $r<P$, where $P$ is the probability of mating conditional upon encounter. $P$ is regarded as a function of the phenotypes of the pair of potential mates. We let $P$ decay with increasing difference, $d$, according to the function:

$$
P=e^{-d^{2}}
$$

In reality, $d^{2}$ will be a weighted combination of several traits and preferences. Let $y_{i}$ be the $i$ th variable in males, and $x_{i}$ be the corresponding variable in females. It is important to stress that the variables involved in males need not be the same as those in females. The correspondence between $x_{i}$ and $y_{i}$ is not determined by what is measured but by what factors contribute to mating preference. Thus, in a particular population, $x_{i}$ might be good looks and $y_{i}$ might be intelligence, if the probability of mating is determined by a combination of male intelligence and female good looks. It is simplest to assume that the $x_{i}$ and $y_{i}$ are standardised to unit variance and introduce for each pair of traits a weighting factor, $w$, such that the weighted squared distance between the mates is:

$$
d^{2}=\sum_{i} w_{i}\left(x_{i}-y_{i}\right)^{2}
$$

Clearly, if $w_{i}=0$ for a particular trait pair then mating is random with respect to these traits since the probability of mating conditional upon encounter is unity as far as these traits are concerned. The weighting factors are assumed to be population constants as a first approximation though it can be argued that individual differences in $w$ can arise. Such second-order effects can only concern us when we have demonstrated effects of the first order.

\section{The simulations}

\section{(i) General considerations}

A large finite population is assumed and encounters are generated at random between males and females. Distances are computed according to (2) above and the probability of mating from (1). For each encounter $r$ is generated and a stable association is assumed to be formed if $r<P$. Otherwise it is assumed that no mating occurs and the individuals are returned to 
the population. When every unmated individual in the population has had one random encounter, one "cycle" of encounters has been completed. After each cycle several events can occur. It can be assumed that there is no further opportunity for mating and the mating population will consist of whatever individuals have already formed stable associations. Alternatively, a further cycle of encounters can be generated by pairing the remaining males and females at random. If the former strategy is adopted there will be more or less intense selection, depending on the number of cycles and the magnitude of the $w_{i}$ 's. If the latter strategy is followed it is likely that encounters will never cease unless the weights are relaxed somewhat because large distances will be generated by a few extreme members of the population. A reasonable approach is to allow the weights to decay with each new cycle, $n$, according to the function:

$$
w_{i},{ }_{n+1}=w_{i},{ }_{n} e^{-k}
$$

If $k$ is zero, the weights remain constant throughout. A large $k$ results in a rapid approach to random mating on the second and subsequent cycles. After any cycle the covariance matrix of individuals' phenotypes can be computed.

\section{(ii) Application to twins}

In the particular application considered here the population was assumed to comprise 1000 pairs of twins and an equal number of potential spouses. Most of the effects of primary interest can be simulated by assuming that mating is a function of two characteristics of each individual, one for which $w_{i}$ is zero, and one for which the weight is not zero. It was assumed, though not of necessity, that the two characteristics were independently distributed among unrelated individuals in the initial population. If mating is assortative for a number of inherited traits these conditions are unlikely to persist for many generations.

The initial population and the first cycle of random encounters were generated simultaneously by producing 1000 eight-variate random vectors from a multivariate normal distribution with arbitrary zero mean vector and identity covariance matrix apart from the two correlations between twins for traits I and II, which are varied to reflect the degree of assumed familial determination of the traits in twins. The structure of the covariance matrix is illustrated in table 1, for simulated data, in which the expected twin correlation was fixed at 0.9 .

The only non-zero covariances in the initial population are those for the component traits in twins which arise because of the genotypic and environmental similarity between twins for the traits involved in mating. The population thus consisted of 2000 potential mating pairs. In all cycles it was assumed that twins did not influence one another in the selection of mates; that is, members of a twin pair were treated quite independently except in the computation of summary statistics when the unique structure of the population was recognised and analysed as it would in practice.

For a given set of data simulated for particular twin correlations, the same population was subjected to differenț strategies of mating in order to determine more precisely the consequences of parameter changes on the identical set of randomly selected individuals. 
TABLE 1

Variances and correlations observed for 1000 pairs of twins $(\rho=0.9)$ and potential mates at the first random encounter*

\begin{tabular}{|c|c|c|c|c|c|c|c|c|c|c|}
\hline & \multirow[b]{3}{*}{ Trait } & \multicolumn{8}{|c|}{ Correlations $\times 100$} & \multirow{3}{*}{$\begin{array}{c}\text { Variance } \\
\times 100\end{array}$} \\
\hline & & \multicolumn{2}{|c|}{ Spouse 1} & \multicolumn{2}{|c|}{ Twin 1} & \multicolumn{2}{|c|}{ Twin 2} & \multicolumn{2}{|c|}{ Spouse 2} & \\
\hline & & I & II & I & II & I & II & I & II & \\
\hline \multirow[t]{2}{*}{ Spouse 1} & I & 100 & 0 & -1 & 1 & 1 & 3 & 3 & 2 & 99 \\
\hline & II & & 100 & 3 & 4 & 4 & 4 & 1 & 8 & 103 \\
\hline \multirow[t]{2}{*}{ Twin 1} & I & & & 100 & -4 & 89 & -2 & 4 & 0 & 97 \\
\hline & II & & & & 100 & -3 & 90 & -6 & 1 & 97 \\
\hline \multirow[t]{2}{*}{ Twin 2} & $\mathrm{I}$ & & & & & 100 & -1 & 4 & 2 & 98 \\
\hline & II & & & & & & 100 & -4 & 2 & 94 \\
\hline \multirow[t]{2}{*}{ Spouse 2} & I & & & & & & & 100 & 1 & 101 \\
\hline & II & & & & & & & & 100 & 108 \\
\hline
\end{tabular}

\section{(a) Example}

The expected covariances of the twins were set to 0.9 for both variables. These values represent what would be found for two highly heritable traits of unit variance measured on monozygotic twins. The observed $8 \times 8$ covariance matrix of the initial population is given in table 1 . It is seen that the observations based on 1000 pairs of twins and random potential mates follow the expected covariance structure very closely. On the first cycle of mate selection $w_{1}$ was set to 0.5 and $w_{2}$ to zero. That is, selection is based on first trait but not the second.

\section{(b) The first cycle}

After the generation of mating pairs, using the method outlined above, 1182 stable associations were formed at the first cycle. At this point, the $2 \times 2$ contingency table representing twins' concordance for mating was computed (table 2). There is striking evidence that spouse selection is reflected in concordance for the presence or absence of mating. Assortative mating, therefore, produces significant concordance for mating between twins when the mating system is resulting in selection. The selection of spouses also leads to a significant change in the covariance structure of the selected population,

TABLE 2

Twin concordance for mating at the first encounter (number of pairs): $\mathrm{w}=0.5$

\begin{tabular}{llccr}
\multirow{3}{*}{ Twin 2} & \multicolumn{2}{c}{ Twin 1 } & \\
\cline { 3 - 4 } & Mated & 374 & 214 & Total \\
& Not mated & 220 & 192 & 588 \\
& Total & 594 & 406 & 1000
\end{tabular}

$$
\chi^{2}(1)=10.47 ; \mathrm{P}<0.01 \text {. }
$$


as is seen in table 3. The table presents the correlations between relatives and the variances for the data summarised in two ways. The upper triangle of the correlation matrix, and the variances in the right-hand column of the table are computed for all complete pairs in the sample. That is, every individual twin who mated successfully was included in the sample, irrespective of his co-twin's success in mating. The lower triangle and the variances on the bottom row of the table, however, reflect only those families of four individuals (two twins and two mates) created when both twins succeed in finding mates. The variances are greatly reduced for the first trait which is directly involved in mate selection. When the sample consists of all twins who mate, regardless of the performance of co-twins, the variance of the first

TABLE 3

Covariance structure of the population of mates formed at the first encounter. Upper triangle and right hand column give correlations and variances based on all pairs formed. Lower triangle and bottom row give corresponding statistics for twin pairs concordant for mating only. All statistics are $\times 100$

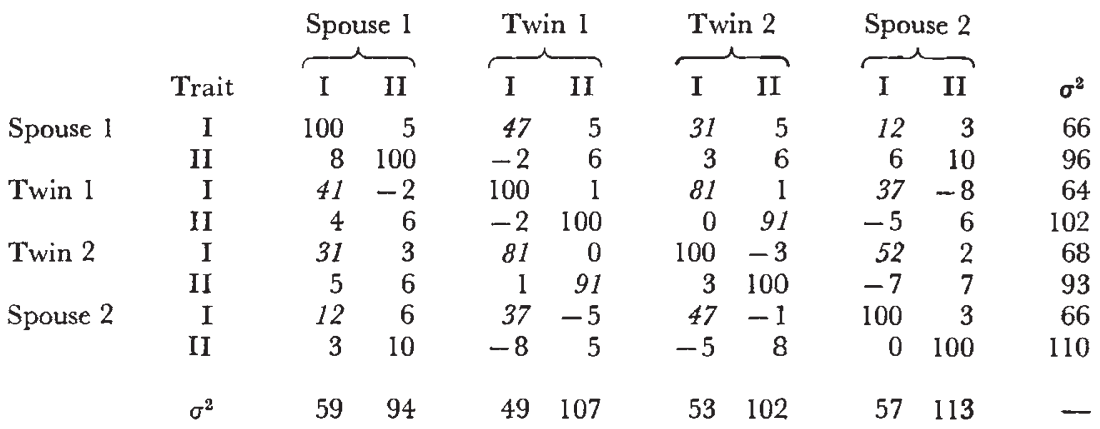

Salient correlations are italicised.

trait in spouses and twins is reduced by approximately one-third as a result of excluding extremes from the sample, by mating preference. When only complete families are considered, however, the reduction in variance is more marked for the twins, being approximately 50 per cent of the original value. The message is clear, if one twin is too extreme to find a mate easily then his co-twin will also have difficulty. Detailed consideration of the correlations will be postponed until later except to note that the correlations are substantial, whilst those for the second trait remain close to zero. The twin correlation for the unselected traits is still close to 0.9 , whereas that for the selected trait has dropped to $0 \cdot 81$, presumably as a result of attenuation due to selection.

\section{(c) Subsequent cycles}

The procedure was continued for further cycles, random encounters being generated between those males and females which had not mated so far, until every individual had mated. In order to ensure that every individual found a mate in a reasonable number of encounters the weight for the first variable was allowed to decay according to (3) above with $k=0 \cdot 1$. After nine cycles, every individual had mated and the correlations in the resulting population 
are given in table 4. Since there is no selection, the variances of twins and spouses for both traits are now close to unity, and the correlations between twins are both close to 0.9 as expected. The marital correlations, i.e., between twins and their own spouses for the first trait, have not altered as a result of further cycles. However, the other correlations involving spouses-those between twins and the spouses of their co-twins and that between twins' spouses-have been increased by continued assortation even though the selection is less intense on further cycles.

\section{TABLE 4}

Covariance structure of population of mates after all individuals have mated. Upper triangle only given since matrix is symmetric*

\begin{tabular}{|c|c|c|c|c|c|c|c|c|c|c|}
\hline & & Spo & se I & Tw & n 1 & $\mathrm{Tw}$ & & Spo & se 2 & \\
\hline & Trait & I & II & I & II & I & II & I & II & $\sigma^{2}$ \\
\hline Spouse 1 & I & 100 & 1 & 46 & 1 & 41 & 5 & 19 & 2 & 104 \\
\hline & II & & 100 & 1 & 2 & 3 & 1 & 4 & 1 & 101 \\
\hline Twin 1 & I & & & 100 & -4 & 89 & -2 & 48 & -2 & 9 \\
\hline & II & & & & 100 & -3 & 90 & -1 & 5 & 9 \\
\hline Twin 2 & I & & & & & 100 & -1 & 53 & 0 & \\
\hline & II & & & & & & 100 & 2 & 5 & \\
\hline Spouse 2 & I & & & & & & & 100 & -1 & 9 \\
\hline & II & & & & & & & & 100 & 11 \\
\hline
\end{tabular}

* Number of pairs formed at successive cycles

\begin{tabular}{lccccccccr} 
Cycle & 1 & 2 & 3 & 4 & 5 & 6 & 7 & 8 & 9 \\
Number of pairs & 1182 & 434 & 188 & 105 & 50 & 23 & 9 & 6 & 3 \\
& \multicolumn{8}{c}{$w=0 \cdot 5 ; k=0 \cdot 1}$.
\end{tabular}

\section{Implications}

Apart from the reduction in variance which results from selection if encounters do not continue until all pairs have mated, there is little difference in the correlational structure of the selected and unselected populations after assortation. The effects of assortation without selection will be considered, therefore, in reasonable confidence that the same remarks will apply to the phenotypic correlations of twins and spouses even if there is selection. This would not be true, of course, for the offspring of the twins.

The reason for including a second variable will become clear when we recognise that the traits we actually measure in a given study may not be those on which assortation takes place directly. Thus although we might measure extraversion in both males and females the trait may actually only be important to one sex. When the trait we measure is a function of the preferences of both sexes we can speak of "symmetric" assortation. When the measured trait is a function of the preferences of only one sex we refer to " asymmetric" assortation. By extracting the appropriate values from the correlation matrix in table 4 it is possible to reproduce the consequences of the different systems of mating for the similarity between twins and their spouses. 


\section{(1) Symmetric assortation}

It is assumed that the measured trait, intelligence say, is trait $I$ in both twins and spouses. The correlations between twins and spouses for intelligence would thus be given by the $4 \times 4$ correlation matrix for trait I as follows:

$\begin{array}{lcccc} & \text { Spouse } & \text { Twin } & \text { Twin } & \text { Spouse } \\ & 1 & 1 & 2 & 2 \\ \text { Spouse 1 } & 1.00 & 0.46 & 0.41 & 0.19 \\ \text { Twin 1 } & 0.46 & 1.00 & 0.89 & 0.48 \\ \text { Twin 2 } & 0.41 & 0.89 & 1.00 & 0.53 \\ \text { Spouse 2 } & 0.19 & 0.48 & 0.53 & 1.00\end{array}$

The twin correlation demonstrates the substantial familial determination of the trait. The marital correlations, 0.46 and 0.53 , would be obtained irrespective of the grouping of subjects into twins. A simple average shows that the degree (approximately 0.5) of assortation generated by the parameters selected is comparable with that found for many human behavioural traits including I.Q. and social attitudes. Correlations which are unique to this design and which promise to justify the analytical power of the approach, are those between a twin and the spouse of his co-twin (0.41 and 0.48$)$ and that between spouses of twins. The average value of the former is approximately 0.45 and equal to the product of the twin and marital correlations. Of particular interest is the spouse correlation of 0.19 which is very close to the product of the twin correlation and the square of the marital correlation. This result demonstrates that a transitive relationship between the marital correlations of relatives is generated by a sensible biological model for the mating system and is contrary to the assertion of the path analytic school, on mathematical rather than biological grounds, that such correlations should not be assumed to be transitive in samples of this type.

If the similarity between twins is due entirely to additive genetic factors, then the correlation of a twin with the co-twin's spouse is equal to the additive genetic correlation between spouses (Fisher's " A ") and the magnitudes of the inequalities: marital correlation > co-twin-spouse correlation $>$ spouse correlation should provide a test of the assumption that assortation takes place on the basis of the measured phenotype, since the three correlations are expected to depend only on $\rho$ and $\mu$. In practice, large samples might be needed to falsify the theory, but such samples are almost certainly going to be smaller than those needed by alternative model-fitting approaches.

\section{(ii) Asymmetric assortation}

If we now suppose that assortation reflects preferences in the twins which are not measured directly but only through their effect on the measured trait of theirspouses, then a different set of expectations are produced. We assume that, for example, we are measuring height in both twins and spouses, but that although individual twins have definite ideas (possibly the result of inherited differences) about the height of a desirable spouse, these have nothing whatever to do with their own height. Trait $I$ in spouses is thus the trait we actually measure (e.g., height) whereas the corresponding trait in twins is not the trait measured but the twins' preference for the trait 
measured. The measured trait in twins is represented by trait II which, in our example, is not under reciprocal selection by potential spouses. The $4 \times 4$ matrix of phenotypic correlations under asymmetric assortation in which twins' preferences are correlated is as follows:

$\begin{array}{lrrrr}\text { Spouse 1 } & 1.00 & 0.01 & 0.05 & 0.19 \\ \text { Twin 1 } & 0.01 & 1.00 & 0.90 & -0.01 \\ \text { Twin 2 } & 0.05 & 0.90 & 1.00 & 0.02 \\ \text { Spouse 2 } & 0.19 & -0.01 & 0.02 & 1.00\end{array}$

The twin correlation has nothing whatever to do with the mating process, as it involves a trait entirely uncorrelated with the underlying (and unmeasured) similarity between twins' preferences with respect to the phenotypes of their spouses. All other correlations are zero, apart from that between spouses which is the product of the correlation between twins' preferences $(\rho=0.9)$ and the square of the unmeasured correlation $(\mu=$ $0.5)$ between twins' preferences and the measured object of their preference.

Thus, in so far as twins and twins alone do the selection, we expect a correlation between the spouses of twins for the trait on which spouse selection is based but we do not expect a marital correlation. In short, if we do not detect a phenotypic correlation between spouses for a single trait it does not necessarily imply mating is random, merely that assortation might be asymmetric.

Clearly, it is senseless to regard assortative mating as the prerogative of twins irrespective of sex. The fundamental asymmetry is not between the preferences of twins and non-twins, but between the preferences of males and females. The above result, therefore, is not expected to be characteristic of twins in general but of twins of one sex. That is, if selection is based primarily on male preferences it will be the spouses of male twins which will display the correlation due to assortative mating. What of the spouses of female twins under such circumstances? The answer lies in the complement of the $4 \times 4$ matrix above. We regard trait $I$ in spouses as the unmeasured scale of preference. It is then assumed that trait II is measured on spouses and trait I on twins. In this case all the correlations with the exception of that between twins are effectively zero, thus:

$\begin{array}{lrrrr}\text { Spouse 1 } & 1.00 & 0.01 & 0.01 & 0.03 \\ \text { Twin 1 } & 0.01 & 1.00 & 0.89 & -0.02 \\ \text { Twin 2 } & 0.01 & 0.89 & 1.00 & 0.00 \\ \text { Spouse 2 } & 0.03 & -0.02 & 0.00 & 1.00\end{array}$

Under asymmetric assortative mating based on phenotype it is inconceivable that the similarity between twins' spouses should be other than confined to the spouses of twins of only one particular sex. If male preferences determine mating, the correlation will be confined to the spouses of male twins. If spouse selection is primarily determined by females, the correlation will be confined to the spouses of female twins.

\section{Effects of Ghanging the Parameters}

\section{(i) Intensity of assortation}

Doubling the weight given to the selected trait at the outset and setting $w=1$ results in the selection of 906 pairs at the first encounter, giving the $43 / 3-\mathrm{H}$ 
twin concordances for mating in table 5. Again, there is some evidence of concordance for mating in twins as a result of assortation. All individuals had mated by the end of the 10th cycle. Table 6 gives the observed covariance structure for the unselected population produced by this point. Exactly the same relationships can be verified for these correlations as were discussed in detail above, except that the correlation between mates is raised from approximately 0.5 to an average of 0.67 , with a resulting elevation of other correlations in the table.

TABle 5

Twin concordance for mating at the first encounter: $\mathrm{w}=1$

\begin{tabular}{|c|c|c|c|c|}
\hline \multirow[b]{3}{*}{ Twin 2} & & \multicolumn{2}{|c|}{ Twin 1} & \multirow[b]{2}{*}{ Total } \\
\hline & & Mated & Not mated & \\
\hline & $\begin{array}{l}\text { Mated } \\
\text { Not mated } \\
\text { Total }\end{array}$ & $\begin{array}{l}221 \\
230 \\
451\end{array}$ & $\begin{array}{l}234 \\
315 \\
549\end{array}$ & $\begin{array}{r}455 \\
545 \\
1000\end{array}$ \\
\hline
\end{tabular}

TABLE 6

Covariance structure of population of mates after all individuals have mated. More extreme assortation $(w=1, k=0 \cdot 1)$

\begin{tabular}{|c|c|c|c|c|c|c|c|c|c|c|}
\hline \multirow{4}{*}{ Spouse 1} & \multirow[b]{2}{*}{ Trait } & \multicolumn{2}{|c|}{ Spouse 1} & \multicolumn{2}{|c|}{ Twin 1} & \multicolumn{2}{|c|}{ Twin 2} & \multicolumn{2}{|c|}{ Spouse 2} & \multirow[b]{2}{*}{$\sigma^{2}$} \\
\hline & & I & II & I & II & I & II & I & II & \\
\hline & I & 100 & 2 & 69 & -1 & 60 & -3 & 39 & -1 & 98 \\
\hline & II & & 100 & 5 & 1 & 4 & 4 & 4 & 0 & 106 \\
\hline \multirow[t]{2}{*}{ Twin 1} & I & & & 100 & -4 & 89 & -2 & 60 & -2 & 97 \\
\hline & II & & & & 100 & -3 & 90 & -1 & 4 & 97 \\
\hline \multirow[t]{2}{*}{ Twin 2} & I & & & & & 100 & -1 & 65 & -1 & 98 \\
\hline & II & & & & & & 100 & -1 & 4 & 93 \\
\hline \multirow[t]{2}{*}{ Spouse 2} & I & & & & & & & 100 & -1 & 100 \\
\hline & II & & & & & & & & 100 & 106 \\
\hline
\end{tabular}

Number of pairs formed at successive cycles

$\begin{array}{lcccccccccc}\text { Cycle } & 1 & 2 & 3 & 4 & 5 & 6 & 7 & 8 & 9 & 10 \\ \text { Number of pairs } & 906 & 483 & 244 & 160 & 101 & 52 & 30 & 14 & 6 & 4\end{array}$

(ii) Twin correlation

The above examples assume a twin correlation of 0.9 for both measurements and preferences. Such values are often found for monozygotic twins in human populations. However, if the analysis of any trait is restricted simply to identical twins it is not possible to disentangle the inherited and cultural components of twin similarity. This applies no less to attempts to separate the genetic and environmental components of assortative mating. Repetition of the above simulations for a population in which the twin correlation is reduced to 0.45 (i.e., half that for $\mathrm{MZ}$ twins) enable us to examine the consequences of assortation in dizygotic twins of like sex for traits which are entirely due to the additive effects of inherited differences. Comparison of these results with those generated on the assumption of a 
higher twin correlation will confirm the view that the study of twins' spouses can indeed produce an effective analysis of the causes of assortative mating. The findings for 1000 pairs of twins are given in table 7 for $w$ set to 0.5 and 1 with a $k$ of $0 \cdot 1$ on the assumption of symmetric assortation. The values expected under asymmetric assortation can be deduced simply by setting the appropriate redundant correlations to zero by analogy with the examples above. The findings accord generally with the prediction that correlations apart from the marital correlation should be reduced by 50 per cent on account of the reduction of the twin correlation. The agreement is especially

TABLE 7

The effect of reducing the expected twin correlation to 0.45 on the pattern of assortation in the absence of selection

\begin{tabular}{ccccccc} 
& No. of & No. mated & \multicolumn{4}{c}{ Correlations } \\
\cline { 2 - 6 } & cycles & on 1st cycle & Twin & Marital & Co twin-spouse & Spouse-spouse \\
0.5 & 8 & 1176 & 0.44 & 0.55 & 0.28 & 0.17 \\
1.0 & 11 & 918 & 0.44 & 0.65 & 0.28 & 0.20
\end{tabular}

close when assortation is more marked. In the case of the simulation involving less intense assortation the marital correlation is a little higher than that found for the comparable case when the twin correlation was $0 \cdot 9$. That is probably due to chance but leads to inflation of the co-twin-spouse and the spouse-spouse correlations.

Acknowledgments. - The work was undertaken while the author was an S.R.C. Advanced Fellow in Genetics at Birmingham University and is part of a programme in the application of biometrical genetics to human variation supported by the M.R.C. Some of this work was conducted during the tenure of an A. D. Williams Distinguished Fellowship in Genetics at the Medical College of Virginia, U.S.A. The author is indebted to Professor W. E. Nance and Professor J. L. Jinks for stimulating discussion.

\section{REFERENCES}

EAVEs, L. J., I.AST, KRYSTYNA, A., Young, P. A., AND MARTin, N. G. 1978. Model-fitting approaches to human behaviour. Heredity, 41, 249-320.

FISHER, R. A. 1918. The correlation between relatives on the supposition of Mendelian inheritance. Trans. Roy. Soc. Edinburgh, 52, 399-433.

NANCE, W. E., AND COREY, L. A. 1976. Genetic models for the analysis of data from the families of identical twins. Genetics, 83, 811-826.

RAO, D. C., MORTON, N. E., AND YEE, S. 1976. Resolution of cultural and biological inheritance by path analysis. Amer. J. Human Genet., 26, 331-359.

WILSON, s. R. 1973. The correlation between relatives under the multifactorial model with assortative mating. I. The multifactorial model with assortative mating. Ann. hum. Genet., 37, 189-204. 